\title{
On the interactions of awareness and certainty
}

\author{
Hans van Ditmarsch ${ }^{1,2}$ and Tim French ${ }^{3}$ \\ 1 Department of Logic, University of Sevilla, Spain, hvd@us.es \\ 2 Institute of Mathematical Sciences Chennai, India \\ 3 Computer Science and Software Engineering, The University of Western Australia, \\ tim.french@uwa.edu.au
}

\begin{abstract}
We examine the interactions of knowledge and awareness in dynamic epistemic logic. Implicit knowledge describes the things that an agent could infer from what is known, if the agent were aware of the necessary concepts. Reasoning techniques that are robust to incomplete awareness are important when considering interactions of automated agents in complex dynamic environments, such as the semantic web. Here we revisit Hector Levesque's original motivation of implicit knowledge and consider several contemporary realizations of implicit knowledge. We present a framework to compare different interactions of knowledge and awareness in the context of public announcements, and introduce a new formalism for tacit knowledge.
\end{abstract}

\section{Introduction}

The term implicit belief was introduced by Hector Levesque [13] to describe "not what an agent believes directly, but what the world would be like if what he believed were true."

Implicit knowledge has come to describe the things that an agent knows, but of which the agent is not fully aware [6]. Implicit knowledge has limited significance with respect to static knowledge and awareness, where explicit knowledge suffices for most applications. However, implicit knowledge is essential for the formalization of dynamic epistemic systems, where the knowledge and awareness of agents changes over time. Implicit knowledge may be used to model latent facts that the agent subconsciously recognizes, inferences that the agent can make, but has not yet made, or aspects of the agent's knowledge that the agent does not yet have the vocabulary to express.

Certainty and awareness are two separate and important facets of knowledge. Certainty describes an agent's confidence in a given scenario, and awareness describes an agent's ability to perceive a given scenario. Epistemic logic is the logic of knowledge and is very much focussed on the element of certainty (or uncertainty). It is very well studied [7] and has applications in reasoning about security in information systems. While epistemic logic presumes logically omniscient agents [6], practical agents are not able to hold all relevant facts on their mind at once, and do not have the computational resources to instantly make all valid inferences from their knowledge base. This has lead to several models of incomplete reasoning applied to human activities (particularly commercial transactions) $[13,14,10]$.

One approach to address these limitations has been to temper an agent's knowledge with an element of awareness. Thus, we can have an agent who is unaware of a proposition (that is, oblivious to its existence), as opposed to simply uncertain as to its interpretation. This is a simple and elegant approach that allows us to differentiate information that an agent explicitly knows from information that may be implicit in the agent's internal state.

In this paper we will examine dynamic interactions between awareness and knowledge. As with dynamic epistemic logic [5] where an agent may learn new facts about the world, we may consider awareness dynamics where an agent may become aware of new properties 
in the world. Becoming aware should leave the agent in an enlightened state, and able to accumulate new knowledge (with respect to the properties of which the agent is newly aware) that is compatible with what the agent already knows. The notion of implicit knowledge is essential to capture this compatibility between knowledge states. We will give a uniform framework to consider these different notions and examine their expressivity in the context of dynamic epistemic operations.

\section{The logics of dynamic knowledge and awareness}

The properties of knowledge and belief in the presence of full awareness have been extensively studied and are well-understood. They are effectively modelled by normal modal operators that are reflexive, transitive and symmetric (for knowledge) or serial and Euclidean (for belief) [7]. However without full awareness things become more complicated. The first issue to arise is that the logic of explicit knowledge is no longer a normal modal logic. For example, if an agent is not aware of the $\varphi=p \vee \neg p$, then $\mathbf{K}_{i} \varphi$ is not true even though $\varphi$ is valid. That is, the necessitation rule fails for explicit knowledge.

\subsection{Language}

We suppose that we are extending a multi-agent propositional epistemic logic, defined over a set of agents $N$ and and set of atomic propositions, $P$. We augment multi-agent epistemic logic with a new operator: $\mathbf{A}_{i} \varphi$, to mean that agent $i$ is aware of all the concepts in $\varphi$. We note, some treatments of knowledge and awareness do not have an explicit operator for awareness, preferring to treat it as an abbreviation. The construct $\mathbf{K}_{i} \varphi$, "agent $i$ knows $\varphi$ " stands in our case for "agent $i$ explicitly knows $\varphi$ ", and we add an additional operator, $\mathbb{K}_{i} \varphi$ to mean "Agent $i$ implicitly knows $\varphi$ ". We also use the notation $[\psi] \varphi$ to mean, "After $\psi$ is announced, $\varphi$ is true". The effect of this public announcement is two-fold. First, it brings $\psi$ to the attention of all agents. Any agent who was previously not aware of $\psi$ or it's constituent parts, now is. Secondly, it informs the agent that $\psi$ is indeed true, so any world the agent considered where $\psi$ is not true (given the newly found awareness) is discounted.

Definition 1 (Language). Given are a countably infinite set of propositional variables (facts) $P$, and a (disjoint) countably infinite set of agents $N$. The language $\mathcal{L}$ is defined as

$$
\varphi::=\top|p| \varphi \wedge \varphi|\neg \varphi| \mathbf{K}_{i} \varphi\left|\mathbb{K}_{i} \varphi\right| \mathbf{A}_{i} \varphi \mid[\varphi] \varphi
$$

where $i \in N$ and $p \in P$. Implication $\rightarrow$, disjunction $\vee$, and equivalence $\leftrightarrow$ are defined by abbreviation.

Finally we will consider a base semantics as a common framework for considering various interactions of knowledge and awareness. The semantics will extend those for multi-agent epistemic (modal) logic with an awareness function that assigns for each agent and each possible world, the set of formulae of which that agent is aware of in that world.

Definition 2 (Epistemic awareness model). An epistemic awareness model for $N$ and $P$ is a tuple $M=(S, R, \mathcal{A}, V)$ that consists of:

- a domain $S$ of (factual) states (or 'worlds');

- an accessibility function $R: N \rightarrow \mathcal{P}(S \times S)$;

- an awareness function $\mathcal{A}: N \rightarrow S \rightarrow \mathcal{P}(\mathcal{L})$; and

- a valuation function $V: P \rightarrow \mathcal{P}(S)$. 
For $R(i)$ we write $R_{i}$ and for $\mathcal{A}(i)$ we write $\mathcal{A}_{i}$; accessibility function $R$ can be seen as a set of accessibility relations $R_{i}$, and $V$ as a set of valuations $V(p)$. A pointed epistemic awareness model $(M, s)$ is an epistemic awareness state.

The awareness function is defined such that $\mathcal{A}_{i}(s)$ is the set of propositions that the agent $i$ is aware of in state $s$, and as such we will require that it is closed under subformulas (so $\varphi \in \mathcal{A}_{i}(s)$ implies $\psi \in \mathcal{A}_{i}(s)$ for all subformulas $\psi$ of $\varphi$ ). In [6] various other closure properties are considered for agent awareness. As we will consider dynamic models it is convenient to denote the elements of the models $M$ as $\left(S^{M}, R^{M}, \mathcal{A}^{M}, V^{M}\right)$, and to denote pointed models $(M, s)$ as $M_{s}$. The conditions on the accessibility function $R^{M}$ may be varied to reflect different interpretations of knowledge and belief. Some semantics are defined with respect to general frame conditions $(\mathbf{K})[13,4]$, some are defined with respect to belief (KD45) [6] and some are defined with respect to knowledge (S5) [14,2]. Furthermore, some semantics are only defined in the instance where a frame has a single accessibility relation $[13,11]$. The semantics presented here are compatible with each of these restrictions.

Definition 3 (Semantics). Let $M=(S, R, \mathcal{A}, V)$ be given, and suppose $s \in S$.

$$
\begin{aligned}
& (M, s) \models \top \\
& (M, s) \models p \quad \text { iff } s \in V(p) \\
& (M, s) \models \varphi \wedge \psi \quad \text { iff }(M, s) \models \varphi \text { and }(M, s) \models \psi \\
& (M, s) \models \neg \varphi \quad \text { iff }(M, s) \not \models \varphi \\
& (M, s) \models \mathbf{A}_{i} \varphi \quad \text { iff } \varphi \in \mathcal{A}_{i}(s) \\
& (M, s) \models \mathbf{K}_{i} \varphi \quad \text { iff } \varphi \in \mathcal{A}_{i}(s) \text { and } \forall t \in s R_{i}, \quad(M, t) \models \varphi \\
& (M, s) \models[\psi] \varphi \quad \text { iff } s \in S^{M^{\psi}} \Rightarrow\left(M^{\psi}, s\right) \models \varphi \quad \text { (see below) }
\end{aligned}
$$

The semantic definition for public announcements requires us to adjust the model to reflect the agents' newly acquired knowledge. On receiving the public announcement that $\psi$ is true, all agents may disregard any state where $\psi$ is not true. Furthermore, they become aware of the formula $\psi$, if they were not already [17], [2] . The updated model is $M^{\psi}$ where

$$
\begin{aligned}
& S^{M^{\psi}}=\left\{s \in S^{M} \mid(M, s) \models \varphi\right\} \\
& R^{M^{\psi}}=R^{M} \cap S^{M^{\psi}} \times S^{M^{\psi}} \\
& \forall i \in N, \forall s \in S^{M^{\psi}}, \mathcal{A}_{i}^{M^{\psi}}(s)=\mathcal{A}_{i}^{M}(s) \cup\left\{\psi^{\prime} \mid \psi^{\prime} \subseteq \psi\right\} \\
& \forall p \in P, V^{M^{\psi}}(p)=V^{M}(p) \cap S^{M^{\psi}} .
\end{aligned}
$$

We will consider several interpretations for implicit knowledge, but in all cases we have that explicit knowledge is simply defined as implicit knowledge plus awareness, so $\mathbf{K}_{i} \varphi \doteq$ $\mathbb{K}_{i} \varphi \wedge \mathbf{A}_{i} \varphi$ (where $\doteq$ is used to define syntactic abbreviations). We define the dual notions of explicit and implicit knowledge as $\mathbf{L}_{i} \varphi$ (agent $i$ explicitly suspects $\varphi$ ) and $\mathbb{L}_{i} \varphi$ (agent $i$ implicitly suspects $\varphi$ ). Note that as it is intuitive that explicit suspicion requires that the agent is aware of $\varphi, \mathbf{L}_{i}$ is not the exact dual of $\mathbf{K}_{i}$, but rather defined as implicit suspicion plus awareness. Consequently we may define $\mathbb{K}_{i}$ and $\mathbf{A}_{i}$ as the only atomic operators, and use the abbreviations: $\mathbf{K}_{i} \varphi \doteq \mathbb{K}_{i} \varphi \wedge \mathbf{A}_{i} \varphi, \mathbb{L}_{i} \varphi \doteq \neg \mathbb{K}_{i} \neg \varphi$, and $\mathbf{L}_{i} \varphi \doteq \mathbb{L}_{i} \varphi \wedge \mathbf{A}_{i} \varphi$

We will give a simple example of how public announcements update the knowledge state of an agent. Suppose that Alice and Bob are ordering a meal at a restaurant. On reading the menu, Bob notes that Chicken and Beef is available, but he does not know which one Alice will order. He is not aware that the soup of the day is pumpkin soup. Alice announces she will have either the chicken or the pumpkin soup. After this exchange Bob knows she will not have the beef, and he becomes aware of the pumpkin soup. This situation in depicted in Figure 1. The three worlds labelled $c, b$ and $p$ correspond to the worlds where Alice orders 
chicken beef or pumpkin respectively, and the atoms each agent is aware of are marked at each world (we assume that agents are aware of all propositions made of these atoms). The agents' knowledge relations are the transitive, reflexive closure of the relations shown. (So the arrow labelled $B o b$ between worlds $\mathbf{c}$ and $\mathbf{p}$ indicates that Bob cannot distinguish the world where Alice orders beef from the world where Alice orders chicken.)

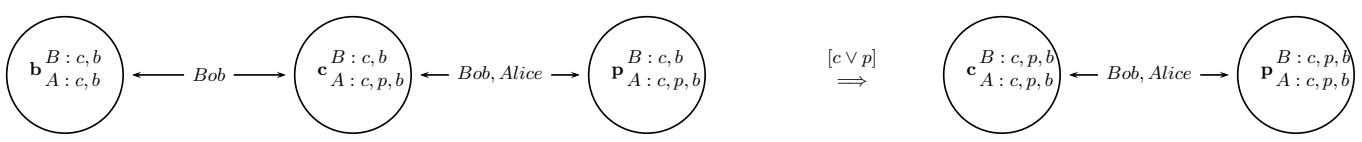

Fig. 1. The scenario before and after Alice makes her announcement.

\section{Implicit knowledge}

In this section we will examine three different variations of implicit knowledge and relate them to existing logics of knowledge, belief and awareness.

\subsection{Strong implicit knowledge}

Levesque's [13] work introduced the concept implicit belief, and was one of the first papers to separate the concerns of awareness and certainty. His response to the problem of logical omniscience was to separate implicit belief from explicit belief. from [13]: "...a sentence is explicitly believed when it is actively held to be true by an agent and implicitly believed when it follows from what is believed". The semantic formulation for this logic is given through situations rather than possible worlds, and for each formula a situation may support the truth of that formula, the falsity of that formula or neither. These situations were potentially incoherent in that a situation may support the truth of both $\varphi$ and $\neg \varphi$ for some formula $\varphi$. This gave a very general approach for agents without logical omniscience. The semantics structures were also kept as general as possible. To define implicit belief, a compatibility relation was required to determine which situations are compatible with the agent's explicit beliefs. An agent implicitly believes a proposition if it is true in all compatible situations.

This approach combines elements of modal logic and situation calculus to reason about the knowledge of imperfect reasoners. A subsequent, and more involved, approach along these lines is given by Cadoli and Schaerf [3] which separate interpretations for situations which are coherent, but incomplete, and those which are complete, but incoherent. See [12] for further discussion and generalizations.

In our setting we present an analogous version of implicit knowledge by refining what is meant by a "compatible situation". Levesque's original version defined compatible to be propositionally consistent with respect to propositions of which the agent was aware. As we are using modal logic we have a well established form of compatibility available, bisimulation, which is known to relate two finite models exactly when they satisfy identical sets of formulas. In [4] the concept of a bisimulation was adjusted to reflect the agent's awareness states.

Awareness bisimulations capture the notion of equivalence in a model, up to an agent's state of awareness. Note that the definition is recursive, so that if agent $A$ considers agent $B$ 's explicit knowledge of $\varphi$, then both agents must be aware of $\varphi$.

Definition 4 (Awareness bisimulation). Suppose we are given epistemic awareness models $M=(S, R, \mathcal{A}, V)$ and $M^{\prime}=\left(S^{\prime}, R^{\prime}, \mathcal{A}^{\prime}, V^{\prime}\right)$. For all subformula-closed $A \subseteq \mathcal{L}$ we say a relation $\mathfrak{B}[A]$ is an $A$-awareness bisimulation iff for all $\left(s, s^{\prime}\right) \in \mathfrak{B}[A]$ : 
atoms for all $p \in A, s \in V(p)$ iff $s^{\prime} \in V^{\prime}(p)$;

aware for all $i \in N, \mathcal{A}_{i}(s) \cap A=\mathcal{A}_{i}^{\prime}\left(s^{\prime}\right) \cap A$;

forth for all $i \in N$, if $t \in S$ and $R_{i}(s, t)$ then there is a $t^{\prime} \in S^{\prime}$ such that $R_{i}^{\prime}\left(s^{\prime}, t^{\prime}\right)$ and $\left(t, t^{\prime}\right) \in \mathfrak{B}\left[A^{i} \cap \mathcal{A}_{i}(s)\right]$;

back for all $i \in N$, if $t^{\prime} \in S^{\prime}$ and $R_{i}^{\prime}\left(s^{\prime}, t^{\prime}\right)$ then there is a $t \in S$ such that $R_{i}(s, t)$ and $\left(t, t^{\prime}\right) \in \mathfrak{B}\left[A^{i} \cap \mathcal{A}_{i}^{\prime}\left(s^{\prime}\right)\right]$.

where $A^{i}=\left\{\psi \subseteq \varphi \mid \mathbf{K}_{i} \varphi \in A\right.$ or $\left.\mathbb{K}_{i} \varphi \in A\right\}$. An epistemic awareness state $\left(M^{\prime}, s^{\prime}\right)$ is

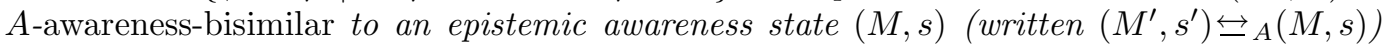
iff $\left(s, s^{\prime}\right) \in \mathfrak{B}[A]$.

Note the bisimulation is given modulo a subformula closed set of propositions, so an agent aware of propositions $P$, would consider structures $M_{s}$ and $M_{s^{\prime}}^{\prime}$ equivalent (as far as he explicitly knows), if they agree on the interpretation of all propositions in $P$. Awareness bisimulations can be seen to be reflexive, symmetric and transitive, so we have the following proposition.

Proposition 1. For all $A \subseteq \mathcal{L}$ A-awareness bisimulation is an equivalence relation.

The strong implicit semantics are given below. Note that as all semantics differ only in their interpretation of implicit knowledge we will only distinguish the implicit knowledge operators $\left(\mathbb{K}^{\mathfrak{I}}\right.$, know $I^{\mathfrak{L}}$ and $\left.\mathbb{K}^{\mathfrak{T}}\right)$. When the semantics of the operator is clear from its context, we will just use $\mathbb{K}$.

Definition 5 (Strong implicit semantics). Let $M=(S, R, \mathcal{A}, V)$ be given. We define

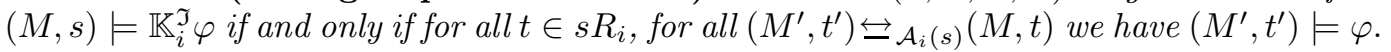

The main innovation in these semantics is the treatment of knowledge. An agent knows $\varphi$ only if in all accessible states $\varphi$ remains true for every possible interpretation of all concepts that she is unaware of. We achieve this by extending the agent's accessibility relation by composing it with bisimulation modulo those concepts of which the agent is unaware.

The following lemma shows the correspondence between strong implicit knowledge and Levesque's original motivation, and may be proven by induction over the complexity of $\varphi$.

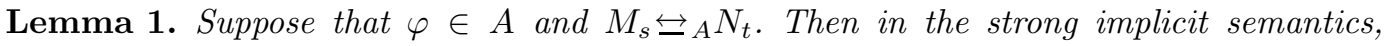
$M_{s} \models \varphi$ iff $N_{t} \models \varphi$.

As an agent aware only of the formulas in $A$ will be unable to distinguish any two models that are $A$-bisimilar it follows that an agent will implicitly know $\varphi$ if and only if $\varphi$ is true in every situation that is indistinguishable to the current situation for that agent.

\subsection{Latent knowledge}

A simpler approach to handling interactions of knowledge and awareness is presented in Fagin's and Halpern's seminal paper [6]. Their approach is to generalize epistemic logic [7] to also account for the agent's awareness, rather than to invent a wholly new approach. Fagin and Halpern presented a generalization of Levesque's approach, where models are as described in Definition 2 and the semantics are as in Definition 3 as well as:

Definition 6 (Latent semantics). Let $M=(S, R, \mathcal{A}, V)$ be given. Then $(M, s) \models \mathbb{K}_{i}^{\mathfrak{L}} \varphi$ if and only if $\forall t \in s R_{i},(M, t) \models \varphi$. 
These semantics present an elegantly simple way to address the gap between uncertainty and unawareness. Implicit knowledge is modelled simply as knowledge with or with out awareness. In essence, the logic presents a syntactic restriction for explicit knowledge (so that knowledge is only meaningfully considered for terms the agent is aware of) and for all other terms implicit knowledge stands as a placeholder for knowledge without awareness.

There were actually three different interpretations of awareness presented in [6], where the approach reported above is the second (the logic of general awareness). The logics presented in [6] are well-defined for multiple agents, nested belief, and do not permit incoherent situations, as Levesque's approach does. However, these practicalities come at a cost. Whereas Levesque's logic of explicit and implicit belief had a clear definition of implicit belief: "a sentence... is implicitly believed when it follows from what is (explicitly) believed"; there is no such such motivation or even definition in the work of Fagin and Halpern.

The elegant simplicity of this approach contrasts the complexity of awareness bisimulations. However, it comes at the expense of having an essential interpretation of implicit knowledge. Indeed the only motivation for implicit belief is given in terms of the abstract semantics: "Implicit belief differs from explicit belief in that for implicit belief we do not take the awareness function into account". Thus we might infer that implicit belief is the belief an agent would have were they fully aware, but there is no understanding given for how this state of parallel awareness might actually manifest itself. For example, $\mathbf{K}_{i}\left(\mathbb{K}_{j}^{\mathfrak{I}} \varphi \wedge \neg \mathbf{K}_{j} \varphi\right)$ describes a situation where agent, $i$ knows that $j$ implicitly knows $\varphi$, and also that $j$ does not explicitly know $\varphi$. This presents a strange situation where other people may know more about what you know than you do. There are various interpretations that could support such a scenario (resource-bounded reasoning, absent-mindedness etc), but there is no general property of knowledge, belief and awareness that matches this notion of implicit knowledge. Rather, it is a convenient semantic device to ensure an agent's explicit knowledge, whilst possibly incomplete, is consistent.

\subsection{Tacit knowledge}

Tacit knowledge is a weaker version of latent knowledge which differs in its treatment of information of which an agent is unaware. It occupies a middle ground between latent knowledge (which describes exactly what an agent should know and should not know) and strong implicit knowledge (which assumes an agent knows nothing that can't be derived from explicit knowledge). The term tacit knowledge was introduced by Polanyi [15] to describe knowledge that was held by someone, but which was very hard to communicate or make explicit. The example of how to ride a bike is often given. Many people know how to ride a bike, but if they were asked to write down exactly how they are able to, they would struggle to share their knowledge.

Tacit knowledge allows for "unspoken knowledge" things an agent can or should know, but of which the agent is not aware. However it does not extend this to tacit ignorance as latent knowledge does. Thus we may permit an agent who does not have the facility of geometry to tacitly know that two distinct parallel lines will never meet, as this is true in every world the agent considers possible (the agent does not consider non-Euclidean geometries possible). The agent is not aware of concepts such as "parallel" so this is not explicit knowledge. Tacit knowledge captures unspoken truths of which an agent is not aware, but which persist nonetheless in all worlds all worlds the agent considers possible. The mechanism by which this is achieved in the the refinement, which is closely related to the bisimulation. 
Definition 7 (Awareness Refinement). Let epistemic awareness models $M=(S, R, \mathcal{A}, V)$ and $M^{\prime}=\left(S^{\prime}, R^{\prime}, \mathcal{A}^{\prime}, V^{\prime}\right)$ be given. For all $A \subseteq \mathcal{L}$ we say the relation $\mathfrak{R}[A] \subseteq S \times S^{\prime}$ is an $A$-awareness refinement iff for all $\left(s, s^{\prime}\right) \in \mathfrak{R}[A]$ :

atoms for all $p \in A, s \in V(p)$ iff $s^{\prime} \in V^{\prime}(p)$;

aware for all $i \in N, \mathcal{A}_{i}(s) \cap A=\mathcal{A}_{i}^{\prime}\left(s^{\prime}\right) \cap A$;

back for all $i \in N$, if $t^{\prime} \in S^{\prime}$ and $R_{i}^{\prime}\left(s^{\prime}, t^{\prime}\right)$ then there is a $t \in S$ such that $R_{i}(s, t)$ and $\left(t, t^{\prime}\right) \in \mathfrak{R}[P]$.

forth for all $i \in N$, if $t \in S$ and $R_{i}(s, t)$ then there is a $t^{\prime} \in S^{\prime}$ such that $R_{i}\left(s^{\prime}, t^{\prime}\right)$ and $\left(t, t^{\prime}\right) \in \mathfrak{R}\left[A^{i} \cap \mathcal{A}_{i}(s)\right]$.

Epistemic awareness state $\left(M^{\prime}, s^{\prime}\right)$ is an A-awareness-refinement of epistemic awareness

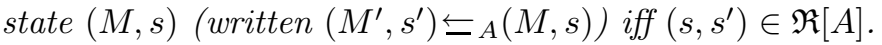

Note that as with Awareness Bisimulation (Definition 4 the Awareness Refinement is restricted to range over models of the logic, so models satisfying the $\mathbf{S 5}$ axioms for epistemic logic, and the KD45 axioms for doxastic logic. It is also interesting to note that the definition of refinement has both the back and forth relations, but they are not symmetrical. The condition back is defined with respect to the full language, so that worlds the agent considers must come from the original model (up to bisimilarity). However forth is defined only with respect to the atoms of which the agent is aware, so the agent may discount some worlds of the original model where it does not affect his explicit knowledge. We then describe tacit knowledge as

Definition 8 (Tacit semantics). Let $M=(S, R, \mathcal{A}, V)$ be given. The semantics are as in Definition 3 and then $(M, s) \models \mathbb{K}_{i}^{\mathfrak{T}} \varphi$ if and only if for all $t \in s R_{i}$, for all $\left(M^{\prime}, t^{\prime}\right)$ where

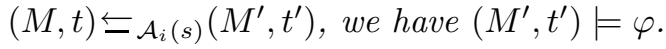

Tacit knowledge is a compromise between rigidity of latent knowledge and the vagueness of strong implicit knowledge. If we consider an agent who is unaware of a proposition, we may not know exactly how an agent may become aware of that proposition, but we may know of some intrinsic relation between that proposition and some other proposition the agent is aware of, which will constrain how an agent may become aware of the proposition. For example an agent observing another agent may know that the second agent tacitly knows summer is hot, even through the second agent is not aware of the concept of summer. As soon as the second agent becomes aware of the concept of summer, the proposition becomes evident. However, if the second agent did not tacitly know summer is hot the first agent still considers it possible that the second agent tacitly knows summer is hot. Although not explicitly stated, tacit knowledge is evident in Heifetz, Meier and Schipper's iterative unawareness [10] which permits a plurality of states of higher awareness for an agent, built upon a set of ground truths, which is the essence of tacit knowledge.

We illustrate the difference between latent, strong implicit and tacit knowledge with the simple example in Figure 2.

\section{Comparative analysis of latent, tacit and strong implicit knowledge}

From now on, we will use the terms strong implicit, tacit, latent and explicit in the context of the definitions above. Negative awareness introspection is the property that an agent 


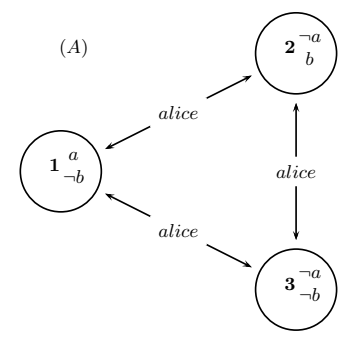

$(B)$

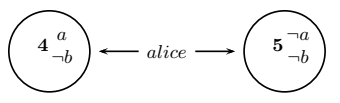

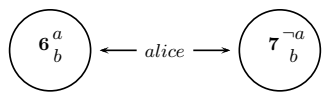

Fig. 2. Suppose that Alice is aware of the atom $a$, but not of $b$. Given the model (A) Alice may latently consider the worlds 1,2 , or 3 possible. However, she may also tacitly consider the worlds 4 and 5 possible in model (B). This is because the worlds 2 and 3 may both be related to the world 5 through a refinement (Alice has no tacit knowledge of $b$ when $a$ is false, but she tacitly knows that if $a$ is true, then $b$ must be false). Finally, Alice may consider the worlds 6 and 7 in model (C) possible in the strong implicit semantics as the interpretation of the atom $b$ is completely unconstrained by the model (A). Note that none of the models change the state of Alice's explicit uncertainty about $a$.

knows when it is not aware of a proposition. We note an interesting distinction between the levels of knowledge that can be seen in the context of negative awareness introspection: latent knowledge does have negative awareness introspection $\left(\models \neg \mathbf{A}_{i} \varphi \rightarrow \mathbb{K}_{i}^{\mathfrak{R}} \neg \mathbf{A}_{i} \varphi\right)$; strong implicit knowledge does not permit negative awareness introspection $\left(\models \neg \mathbf{A}_{i} \varphi \rightarrow \neg \mathbb{K}_{i}^{\mathfrak{I}} \mathbf{A}_{i} \varphi\right)$; and for tacit knowledge negative awareness introspection is satisfiable, but not valid (so neither $\models \neg \mathbf{A}_{i} \varphi \rightarrow \mathbb{K}_{i}^{\mathcal{T}} \mathbf{A}_{i} \varphi$ nor $\models \neg \mathbf{A}_{i} \varphi \rightarrow \neg \mathbb{K}_{i}^{\mathcal{T}} \mathbf{A}_{i} \varphi$ are true).

We consider the application of the various notions of knowledge, by considering a limited form of awareness change. We show how public announcements allows us to express the potential knowledge of an agent, and how this can be related to the levels of knowledge we have described.

Latent knowledge When an agent, $i$, becomes aware of a proposition $\varphi$ through a public announcement, they acquire a knowledge state of the propositions in $\varphi$ that is equivalent to its latent knowledge of these propositions with respect to $\varphi$. That is: $M, s \models[\varphi] \mathbf{K}_{i} \psi$ if and only if $M, s \models \mathbb{K}_{i}^{\mathfrak{R}}(\varphi \rightarrow \psi)$.

Tacit knowledge When an agent, $i$, receives a public announcement, $\varphi$, they learn $\varphi$ is true and also become aware of the subformulas of $\varphi$. Their knowledge of these subformulas is equivalent to their latent knowledge of $\varphi$. However, the agent $i$ would not tacitly know this to be true before the public announcement. Rather they would (tacitly) know that on becoming aware of $\varphi$ they would acquire a knowledge state that is consistent with their explicit knowledge, and one which preserves their tacit knowledge.

Strong implicit knowledge When an agent $i$, receives a public announcement, $\varphi$, they learn $\varphi$ is true, and become aware of the subformulas of $\varphi$. Their knowledge of these subformulas is described by the set of worlds the agent considers possible, as with latent knowledge. However, as with tacit knowledge the agent would not know this prior to the announcement. The agent implicitly knows that becoming aware of $\varphi$, could lead to any new knowledge that is consistent with their current (explicit) knowledge state.

The following lemma establishes the relative strength of each type of knowledge. 
Lemma 2. Let $(M, s)$ be a pointed awareness model, and $\varphi$ be a formula. Then:

$$
\begin{aligned}
& M, s \models \mathbf{K}_{i} \varphi \Longrightarrow M, s \models \mathbb{K}_{i}^{\mathfrak{I}} \varphi \\
& M, s \models \mathbb{K}_{i}^{\mathfrak{I}} \varphi \Longrightarrow M, s \models \mathbb{K}_{i}^{\mathfrak{T}} \varphi \\
& M, s \models \mathbb{K}_{i}^{\mathfrak{T}} \varphi \Longrightarrow M, s \models \mathbb{K}_{i}^{\mathfrak{Q}} \varphi
\end{aligned}
$$

Proof For the first implication it is sufficient to note that if the agent explicitly knows $\varphi$, then the agent is aware of $\varphi$, and so in the context of $\varphi$ the awareness bisimulation is simply a bisimulation (see Definition 4). As bisimulations preserve the interpretation of modal formulas it follows that the agent implicitly knows $\varphi$.

If the agent implicitly knows $\varphi$ then in every accessible world $t \in s R^{M}$, every model $N_{u}$ that is $\mathcal{A}_{i}(s)$ bisimilar to $M_{t}$, we have $N_{u} \models \varphi$. Now by Definitions 4 and 7 any model $N_{v}^{\prime}$ that is a $\mathcal{A}_{i}(s)$ refinement of $M_{t}$, is also a $\mathcal{A}_{i}(s)$ bisimulation of $M_{t}$, and thus $N_{v}^{\prime} \models \varphi$, so $M_{s} \models \mathbb{K}_{i}^{\mathfrak{T}} \varphi$. Finally if $M_{s} \models \mathbb{K}_{i}^{\mathfrak{T}} \varphi$ it follows that $M_{s} \models \mathbb{K}_{i}^{\mathfrak{L}} \varphi$ as the awareness refinement relation is clearly reflexive.

Lemma 3. For all variations of implicit knowledge, $\mathbb{K}_{i} \varphi \wedge \mathbf{A}_{i} \varphi$ is satisfied by a pointed model if and only if $\mathbf{K}_{i} \varphi$ is.

Proof This can be seen by noting in the case of both awareness refinement and awareness bisimulations, when the agent is fully aware of a formula $\varphi$, with respect to $\varphi$ both relations are the same as a bisimulation, which preserves the interpretation of modal formulas.

\section{$5 \quad$ Related approaches}

Here we mention some additional work on awareness. Although these contributions do not add to the variety of semantics for interpreting awareness and implicit knowledge, they certainly provide an appropriate context to consider the advantages and disadvantages of each approach. Hill [11] has examined the dynamics of awareness in the single agent case.

Agotnes and Alechina [1] have considered an expressive extension of logics of knowledge and awareness that allow us to quantify over formulas an agent is unaware of. Particularly, this allows an agent to know another agent knows something that the first agent does not. This expressive ability is also considered by Halpern and Rego [9].

Sillari [16], has also examined the question of knowledge of unawareness, and addresses this question in the context of epistemic first order logic. Also, strong implicit knowledge has previously been examined and axiomatized in [4].

A novel approach to distinguishing what an imperfect agent might know as compared to a logically omniscient agent can be found in $[18,2]$. Here a dynamic epistemic logic is used to model deductive step an agent makes, so we might find an agent who is able to deduce a fact and may indeed be in the process of deducing a fact, but has not yet come to the final realization that the fact is true. We note that [18] gives an axiomatization for such awareness change in the multi-agent case.

In [8] Grossi and Velazquez expand on these ideas using the ideas of multi- valued logic. They differentiate between formulas agents are aware of and formulas agents have access to, such that access is stronger than awareness and captures the idea that a deduction has occurred that has bought the truth of a formula to the attention of an agent, and thus the agent may proceed to use this formula in future deductions.

Heifetz, Meier and Schipper [10] presented a complete framework for describing the interactive knowledge of multiple agents with differing levels of awareness. By interactive 
knowledge, we mean one agent may know about the knowledge and awareness state of another agent. This was an extension of Modica's and Rustichini's theory of unforeseen contingencies [14].

\section{Conclusion and future work}

In this paper we have presented a comparative analysis of the way that awareness and knowledge may interact in dynamic settings. This is an important analysis as different situations will require different types of knowledge and awareness change: modelling secure communications may suit latent knowledge; modelling commercial transactions may suit tacit knowledge; and modelling automated reasoning may be better suited to strong implicit knowledge. The semantic model for tacit knowledge presented here is particularly interesting as tacit knowledge is a well known concept of epistemiology, but while strong implicit and latent forms of knowledge have been previously examined in the context of modal logic, tacit has not.

\section{References}

1. Agotnes, T., Alechina, N.: Full and relative awareness: a decidable logic for reasoning about knowledge of unawareness. In: Proceedings of TARK XI. pp. 6-14. ACM, New York, NY, USA (2007)

2. van Benthem, J., Velazquez-Quesada, F.: Inference, promotion and the dynamics of awareness. In: Knowledge, rationality and action (2009)

3. Cadoli, M., Schaerf, M.: Approximate reasoning and non-omniscient agents. In: TARK (1992)

4. van Ditmarsch, H., French, T.: Becoming aware of propositional variables. In: Banerjee, M., Seth, A. (eds.) Logic and Its Applications - 4th Indian Conference, ICLA 2011. Proceedings. 204-218. (2011)

5. van Ditmarsch, H., van der Hoek, W., Kooi, B.: Dynamic Epistemic Logic, Synthese Library, vol. 337. Springer (2007)

6. Fagin, R., Halpern, J.: Belief, awareness, and limited reasoning. Artificial Intelligence 34(1), 39-76 (1988)

7. Fagin, R., Halpern, J., Moses, Y., Vardi, M.: Reasoning about Knowledge. MIT Press, Cambridge MA (1995)

8. Grossi, D., Velázquez-Quesada, F.: Twelve Angry Men: A study on the fine-grain of announcements. In: He, X., Horty, J., Pacuit, E. (eds.) Logic, Rationality, and Interaction. Proceedings of LORI 2009. 147-160 (2009)

9. Halpern, J., Rego, L.: Reasoning about knowledge of unawareness. Games and Economic Behavior $67(2), 503-525$ (2009)

10. Heifetz, A., Meier, M., Schipper, B.: Interactive unawareness. Journal of Economic Theory 130, 78-94 (2006)

11. Hill, B.: Awareness dynamics. Journal of Philosophical Logic 39, 113-137 2010

12. K.M.Sim: Epistemic logic and logical omniscience ii: A unifying framework. International Journal of Intelligent Systems 15 (2000)

13. Levesque, H.: Logic of implicit and explicit belief. In: Proceedings of AAAI'84. 198-202 (1984)

14. Modica, S., Rustichini, A.: Unawareness and partitional information structures. Games and Economic Behavior 27, 265-298 (1999)

15. Polanyi, M.: The logic of tacit inference. Philosophy 41, 1 (1966)

16. Sillari, G.: Quantified logic of awareness and impossible possible worlds. Review of Symbolic Logic 1(4), 514-529 (2008)

17. Velázquez-Quesada, F.R.: Inference and update. Synthese (Knowledge, Rationality and Action) 169(2), 283-300 (Jul 2009)

18. Velazquez-Quesada, F.: Small steps in dynamics of information. Ph.D. thesis, University of Amsterdam (2011), iLLC Dissertation Series DS-2011-02 